\title{
Charmonium from Statistical Hadronization of Heavy Quarks - a Probe for Deconfinement in the Quark-Gluon Plasma
}

\author{
P. Braun-Munzinger ${ }^{\mathrm{a}, \mathrm{b}}$, J. Stachel $^{\mathrm{c}}$ \\ ${ }^{a}$ ExtreMe Matter Institute EMMI, GSI Helmholtz Zentrum für \\ Schwerionenforschung, D-64291 Darmstadt, Germany \\ b Technical University Darmstadt, D-64289 Darmstadt, Germany \\ ${ }^{\mathrm{c}}$ Physikalisches Institut der Universität Heidelberg, D-69120 Heidelberg, Germany
}

\begin{abstract}
We review the statistical hadronization picture for charmonium production in ultrarelativistic nuclear collisions. Our starting point is a brief reminder of the status of the thermal model description of hadron production at high energy. Within this framework an excellent account is achieved of all data for hadrons built of $(u, d, s)$ valence quarks using temperature, baryo-chemical potential and volume as thermal parameters. The large charm quark mass brings in a new (non-thermal) scale which is explicitely taken into account by fixing the total number of charm quarks produced in the collision. Emphasis is placed on the description of the physical basis for the resulting statistical hadronization model. We discuss the evidence for statistical hadronization of charmonia by analysis of recent data from the SPS and RHIC accelerators. Furthermore we discuss an extension of this model towards lower beam energies and develop arguments about the prospects to observe medium modifications of open and hidden charm hadrons. With the imminent start of the LHC accelerator at CERN, exciting prospects for charmonium production studies at the very high energy frontier come into reach. We present arguments that, at such energies, charmonium production becomes a fingerprint of deconfinement: even if no charmonia survive in the quark-gluon plasma, statistical hadronization at the QCD phase boundary of the many tens of charm quarks expected in a single central $\mathrm{Pb}-\mathrm{Pb}$ collision could lead to an enhanced, rather than suppressed production probability when compared to results for nucleon-nucleon reactions scaled by the number of hard collisions in the $\mathrm{Pb}-\mathrm{Pb}$ system.
\end{abstract}

\section{Introduction}

Investigation of hadron production in ultra-relativistic nucleus-nucleus collisions has revealed convincing evidence for a thermal production mechanism. In particular, the study of yields of hadrons composed of light $(\mathrm{u}, \mathrm{d}, \mathrm{s})$ valence quarks from AGS up to RHIC energies has shown $[1,2,3,4,5,6,7,8,9,10,11,12$ that hadron multiplicities can be described 
quantitatively in the framework of a hadro-chemical equilibrium approach. Within this model the only parameters are thermal quantities: the chemical freeze-out temperature $T$, the chemical potentials $\mu$ and, if applicable, the fireball volume $V$; for a recent review see [13].

The underlying picture of the evolution of the system formed in a collision between two heavy nuclei at high energies is the following: In the early phase of the collision partons are liberated in hard collisions describable by perturbative quantum chromo dynamics (QCD). The partonic system subsequently equilibrates, i.e. reaches (approximate) local momentum isotropy, all the while expanding in beam direction with velocity of light. This system is called the fireball and is characterized by thermal parameters such as a temperature and by an equation of state. Eventually also transverse expansion builds up. The expanding fireball cools with its temperature dropping as $\mathrm{T} \propto \tau^{-1 / 3}$ (or slightly faster due to transverse expansion). Eventually the fireball reaches the phase boundary between quarkmatter and hadronic matter and the partonic degrees of freedom are converted into hadronic degrees of freedom. The corresponding reduction in degrees of freedom is more than a factor of 3 and therefore the volume has to grow accordingly during hadronization. At some temperature equal or below the critical temperature hadron yields are frozen in. This is what is called the chemical freeze-out and the corresponding thermal parameters $\mathrm{T}$ and $\mu$ are determined from the analysis of hadron yields as discussed in the previous paragraph 1 . The now hadronic fireball may expand and cool further until elastic collisions seize to change the momentum distributions. This point is called thermal freeze-out. After this point there may still be some residual interactions (e.g. Coulomb interaction) and weak decays. The resulting momenta and particle types are measured in the detectors.

An important outcome of the hadron yield investigations is that the extracted temperature values rise sharply with energy for energies in the c.m. system per colliding nucleon pair $\sqrt{s_{N N}}$ below $10 \mathrm{GeV}$ and reach constant values near $T=160 \mathrm{MeV}$; this appears to be the limiting temperature for a hadronic fireball. The baryo-chemical potential exhibits a strong (monotonous) decrease as a function of energy. Even detailed features such as the, up to very recently considered rather mysterious, sharp maximum in the excitation function of the $\mathrm{K}^{+} / \pi^{+}$ratio at SPS energies ("the horn") can be described quantitatively if full account is taken of the hadronic mass spectrum [14]. The limiting temperature implies a connection to the QCD phase boundary and it was, indeed, argued [15] that the quark-hadron phase transition drives the equilibration dynamically, at least for SPS energies and above.

The success in describing the production of hadrons containing light quarks in a thermal framework raises the question whether hadrons containing charm (or even bottom) quarks could also be produced thermally. The answer at first glance is no: the charm quarm mass $m_{c} \approx 1.3 \mathrm{GeV}$ brings in a new scale which exceeds significantly any conceivable temperature which may be reached in nucleus-nucleus collisions. It also is much larger than $\Lambda_{Q C D}$, implying that charm production should be describable in a perturbative QCD framework. Thermal production of charm quarks is thus at best (at LHC energy) a small perturbation compared to production in hard scattering, and at presently available (RHIC

1 We have no direct evidence that the quark-gluon plasma before hadronization is in chemical equilibrium although this is likely the case for the light quark flavors and maybe also between light quarks and gluons. 
and SPS) energies completely negligible. It is thus not surprizing that hadrons containing charm quarks, and in particular charmonia were considered key probes to diagnose the fireballs formed in ultra-relativistic nucleus-nucleus collisions.

Charmonia are bound states of charm quarks and their antiquarks. In nuclear collisions at high energy, charm quarks can be produced rather copiously, leading in turn to significant cross sections for charmonium production. The typical radii of charmonium mesons are of order 0.2 - $0.5 \mathrm{fm}$, i.e. comparable to or larger than the mean distance between partons in a Quark-Gluon Plasma (QGP) of temperature of a few hundred MeV. Because of this fact, charmonium production is considered an important probe to determine the degree of deconfinement reached in the fireball produced in ultra-relativistic nucleus-nucleus collisions. In fact, Matsui and Satz argued in [16] that the expected high density of gluons in a QGP formed by collisions between heavy nuclei at ultra-relativistic energy should destroy any charmonia formed prior to the QGP in a process analoguous to Debye screening in an electromagnetic plasma. Consequently, suppression of charmonia (compared to their production in the absence of QGP) was proposed as a "smoking gun" signature for plasma formation in nuclear collisions at high energy. Measurements at the CERN SPS accelerator indeed provided evidence for such suppression [17] in central collisions between heavy nuclei, while no suppression was found in more grazing collisions or collisions between light nuclei, where plasma formation is not expected. However, absorption of charmonium in the nuclear medium as well as its break-up by hadrons produced in the collision were also identified as possible mechanisms leading to charmonium suppression even in the absence of QGP formation [18,19,20,21], and the interpretation of the SPS data remains inconclusive. Recent measurements performed at the RHIC accelerator yielded a $J / \psi$ suppression similar to that observed at the SPS, leading to further puzzles. For a recent review see [22].

Furthermore, it was noted in 23] that charmonium and, in particular, $\mathrm{J} / \psi$ production in $\mathrm{Pb}-\mathrm{Pb}$ collisions at SPS energy exhibit thermal features. Considering these and the general success of the thermal model in describing hadron production, but keeping in mind the new scale brought into the problem by the charm quark mass, led us to propose, in 2000, the statistical hadronization model [24]. In this approach, the number of charm quarks plus their antiparticles is effectively decoupled from the thermal description of charm quark hadronization at the QCD phase boundary, where hadrons are formed from charm quarks with statistical weights calculated at chemical freeze-out. In [24] we have assumed that all charm quarks are formed in initial hard collisions but other processes such as thermal production in extremely hot fireballs formed at LHC energy [25] could be considered in addition. Apart from the total number of charm quarks, the only parameters entering the statistical hadronization model are the thermal parameters which are very well constrained by chemical freeze-out analyses.

The idea of statistical hadronization led to a series of investigations of $J / \psi$ production based on this approach [26,27,28,29,30,31,32]. Initial interest focussed on the available SPS data for $J / \psi$ production in $\mathrm{Pb}-\mathrm{Pb}$ collisions, but the trends for RHIC and LHC were also investigated [313233]. We note that a different approach, based on a kinetic model [34,35,36] has been developed. Here, charmonia are continously formed (and destroyed) during the plasma phase and dynamical input, such as $\mathrm{J} / \psi$ production cross sections, is needed to make predictions. Recently the statistical hadronization model was extended 
to the production of $\Upsilon$ mesons [37,31] and multiply heavy-flavored hadrons [38]. Very comprehensive reviews of quarkonium production in heavy ion collisions can be found in [39, 40].

A key feature of the statistical hadronization model as well as of the kinetic model is the fact that yields for the production of charmonia scale, because the charmonia are combined from charm and anti-charm quarks, with the square of the number of charm quarks $N_{c}$ in the system. This implies that, for sufficiently large $N_{c}$, the production of charmonia should be enhanced [24,2913132] rather than suppressed in a fully deconfined QGP.

An important aspect of charmonium production and its possible modification in nuclear collisions is the sequence of time steps and scales involved in the process. In the original proposal [16] charmonium (or, at least, its precursors, such as a color-octet $c \bar{c}$ state) is produced early, before quark-gluon plasma formation and also in a time interval which is short compared to the collision time. Conversely, in the statistical hadronization model, charmonia and all charmed hadrons are formed late, at the time of hadronization of the QGP. In view of these wildely different approaches a thorough review of the relevant time scales was performed recently [41] and will be important input for the understanding of charmonium as a probe for deconfinement in ultra-relativistic nucleus-nucleus collision.

We start this review with a brief reminder, in section 2, of the current status of the thermal model for hadron production. An account of the statistical hadronization model for charmonia and charmed hadrons is given in section 3. In this section we will also discuss the various time scales relevant for charm, charmonium, and open charm hadron production and discuss their relevance for the applicability of the statistical hadronization model as well as for the study of possible medium effects in the charm sector. Section 4 will deal with the current status of charmonium production in nucleus-nucleus collisions at SPS and RHIC energies and its interpretation in terms of the statistical hadronization model. Our results on open charm and charmonium production and their sensitivities to possible in-medium modifications, in particular at energies below top SPS energy, will be presented in section 5. Section 6 will be devoted to predictions for LHC energy.

\section{Status of thermal production of hadrons in relativistic nucleus-nucleus col- lisions}

Hadron production and in particular hadron yields have been studied with high precision in central nuclear collisions at AGS, SPS, and RHIC energies. These yields can be described by assuming that all hadrons are formed when the fireball reaches a certain temperature T, chemical potential $\mu_{2} 2$, and volume. Under these conditions the yields can simply be described by the thermodynamical grand canonical (or in special cases canonical) ensemble. The underlying process is called "chemical equilibration" in analogy to particle production in the early universe. Recent detailed analyses have been performed by [10,11,14, a comprehensive review can be found in [13. Importantly, the data at SPS and RHIC energies comprise yields of multi-strange hadrons including the $\Omega$ and

\footnotetext{
2 Other chemical potentials are fixed by conservation laws and are thus no free parameters.
} 
$\bar{\Omega}$ baryons. Their yields agree very well with the chemical equilibrium calculation and are strongly enhanced as compared to observations in pp collisions. This implies that, at chemical freeze-out, the strangeness sector is in equilibrium as well.
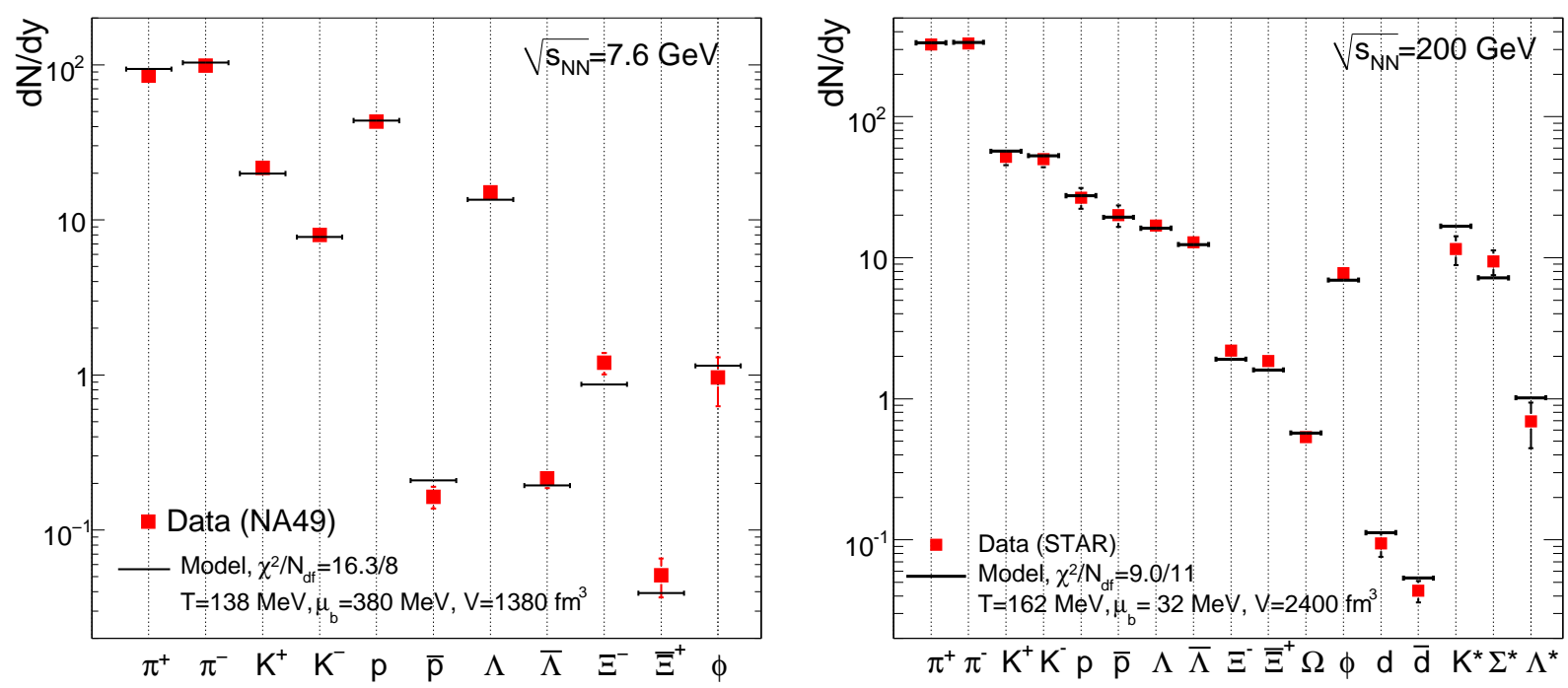

Fig. 1. Experimental hadron yields and model calculations for the thermal parameters of the best fit at the c.m. energies per colliding nucleon pair of 7.6 (left panel) and $200 \mathrm{GeV}$ (right panel). The strongly decaying resonances were not included in the fit.

In Fig. 1 we present a comparison of measured and calculated hadron yields at the energies of $\sqrt{s_{N N}}=7.6 \mathrm{GeV}$ (beam energy of $30 \mathrm{~A} \mathrm{GeV}$ at the SPS) and $\sqrt{s_{N N}}=200 \mathrm{GeV}$. The results are taken from the most recent analysis of [14]. The data sets are very well reproduced by the model calculations and this applies to all energies, from the lowest AGS beam momentum of $2 \mathrm{~A} \mathrm{GeV}$ up to the top RHIC energy of $\sqrt{s_{N N}}=200 \mathrm{GeV}$.

The energy dependence of the so-obtained "chemical" parameters $\mathrm{T}$ and $\mu_{b}$ is shown in Fig. 2. In contrast to the smoothly decreasing chemical potential the temperature parameter first increases strongly with energy but saturates rather abruptly near an energy of $\sqrt{s_{N N}}=10 \mathrm{GeV}$ at a value slightly above $160 \mathrm{MeV}$. This behavior strongly supports Hagedorn's limiting temperature hypothesis [42] and lends support to the notion that the saturation behavior is caused by the QCD phase boundary which, according to lattice QCD calculations, runs for $\mu_{b} \leq 300 \mathrm{MeV}$ at an approximately constant temperature.

The impressive agreement shown in Fig. 1 1 extends also to rather delicate features of hadron production such as the energy dependence of the $K^{+} / \pi^{+}$and $\Lambda / \pi^{+}$ratios as demonstrated in [14]. For the purpose of the present review we note that the thermal parameters $T$ and $\mu_{b}$ as well as the volume which serve as input into statistical hadronization model calculations are completely determined at all energie: 3 . Parametrizations for both are given in [14].

To emphasize the connection to the QCD phase transition we present the values of $T$ and $\mu_{b}$ obtained from our fits of the experimental data in a phase diagram of hadronic matter in Fig. 3. An important observation about the phase diagram is that, for the $40 \mathrm{~A} \mathrm{GeV}$

3 For LHC energy we assume $T=164 \mathrm{MeV}$ and $\mu_{b}=1 \mathrm{MeV}$ in obvious extrapolation of the SPS and RHIC data. 


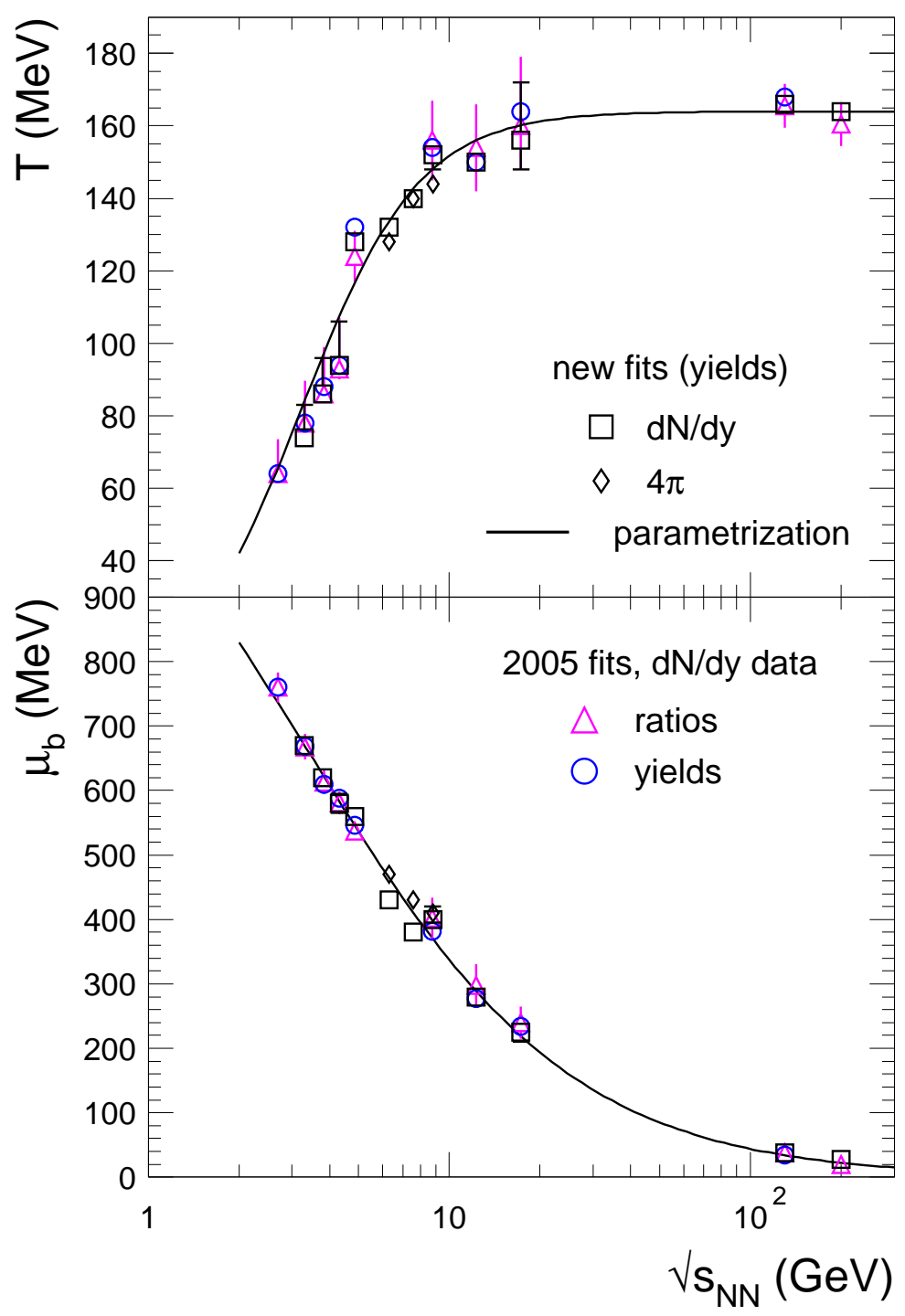

Fig. 2. The energy dependence of temperature and baryon chemical potential for hadron formation. The most recent results (open squares and diamonds) are compared to the values obtained in an earlier study [11] (open triangles and circles). The lines are parametrizations of the most recent results for $T$ and $\mu_{b}$. Figure taken from [14].

SPS energy and above, the thermal parameters for hadron formation agree with the phase boundary calculations from lattice QCD (LQCD) [43,44,45], implying that hadron yields are frozen near the phase boundary.

The LQCD calculation [43] shown in Fig. 3] is for two light quarks $(u, d)$ with realistic (close to physical) masses and a heavy strange quark. The critical temperature at $\mu_{b}=0$ from LQCD calculations is about $170 \mathrm{MeV}$ [46,47] (and refs. therein), with a scale uncertainty of the order of $10 \mathrm{MeV}$ [46,45] but with significany systematic errors [46,47].

Despite significant advances in high performance computing many aspects of the QCD phase transition remain unclear. While the authors of [48] argue that the transition is 
of cross-over type, implying the existence of a critical point, this is disputed on very general grounds in [49] (see also [50] for a general survey). Furthermore, precise values for the transition temperature do not exist today. In fact, results ranging from 150 to 190 $\mathrm{MeV}$ have been obtained for $T_{c}$. The situation is well summarized in [47,51]; according to these authors the range in temperatures could be due to a cross-over nature of the phase transition for small values of $\mu_{b}$ [47]. Here, we note that a large gap between $T_{c}$ and the chemical freeze-out temperature $T$ with a high density hadronic phase in between would make it very difficult to understand why all hadrons freeze-out at the same temperature $T$.

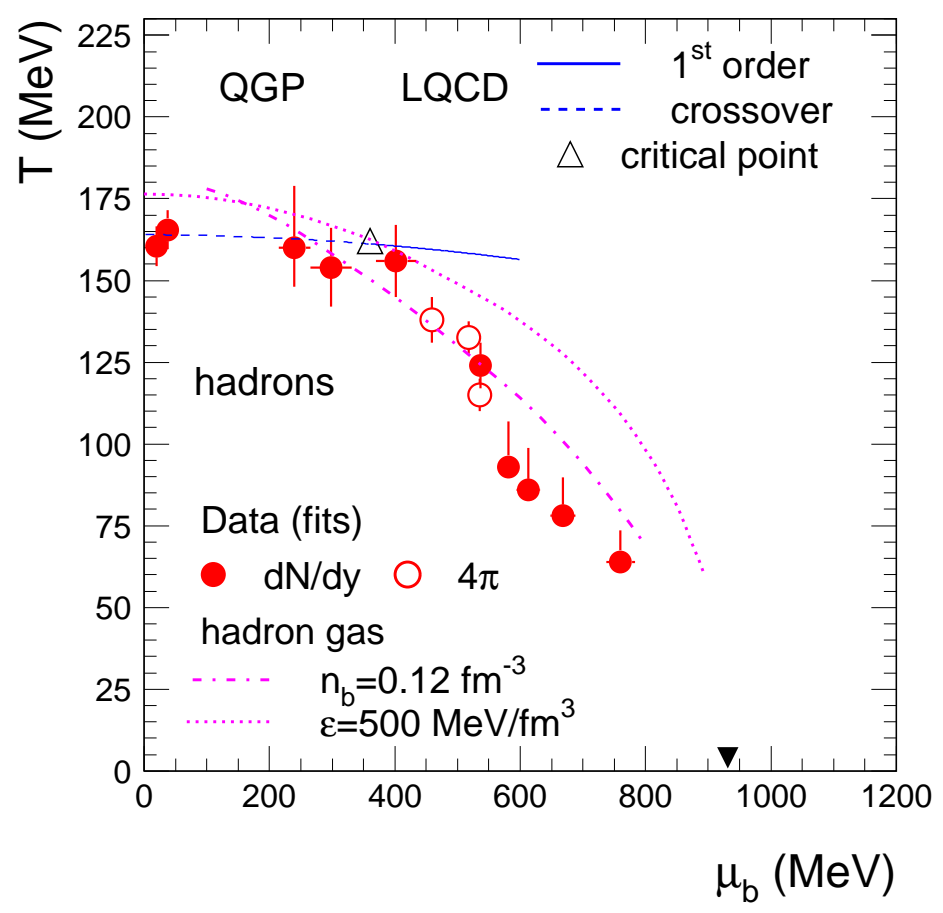

Fig. 3. The phase diagram of nuclear matter in the $T-\mu_{b}$ plane. The experimental values for the chemical freeze-out are shown together with results from lattice QCD calculations [43. The critical point found in the LQCD calculation is marked by the open triangle. Also included are calculations of freeze-out curves for a hadron gas at constant energy density $(\varepsilon=500$ $\mathrm{MeV} / \mathrm{fm}^{3}$ ) and at constant total baryon density $\left(n_{b}=0.12 \mathrm{fm}^{-3}\right)$. The full triangle indicates the location of ground state nuclear matter (atomic nuclei). Figure taken from [11].

Also included in Fig. 3 are calculations of freeze-out curves for a hadron gas at constant energy density $\left(\varepsilon=500 \mathrm{MeV} / \mathrm{fm}^{3}\right)$ and at constant total baryon density $\left(n_{b}=0.12 \mathrm{fm}^{-3}\right)$ [52. The LQCD phase boundary shown in Fig. 3 is apparently departing at large $\mu_{b}$ from our calculated curve of constant energy density, in contrast to the (LQCD-based) results of ref. [44] (see also [53]). However, the errors for the critical line from LQCD calculations become rather significant for increasing $\mu_{b}$ [44.

The freeze-out points which are departing from the LQCD phase boundary are reasonably well described by the curve of a hadron gas at constant baryon density. We note that this curve is very similar to the curve corresponding to an average energy per average number of hadrons of approximately $1 \mathrm{GeV}[54$.

The underlying assumption of the thermal model used to extract the $\left(T, \mu_{b}\right)$ values is (chemical) equilibrium at chemical freeze-out. A natural question then is how is equilibrium achieved? The answer obviously cannot come from within the framework of the thermal model. Considerations about collisional rates and timescales of the hadronic fireball expansion imply that at SPS and RHIC the equilibrium cannot be established in a 
hadronic medium below the critical temperature $T_{c}$ [55/56,57,15]. In a recent paper [15] many-body collisions near $T_{c}$ were investigated as a possible mechanism for the equilibration during hadronization. There it is argued that, because of the rapid density change near a phase transition, such multi-particle collisions provide a natural explanation for the observation of chemical equilibration at RHIC energies and it is suggested that $T=T_{c}$ to within an accuracy of a few $\mathrm{MeV}$.

One should note, however that, for $\mathrm{e}^{+} e^{-}$collisions, analyses of hadron multiplicities [58,59] yield also a thermal-like pattern, suggesting temperature values in the range of $160 \mathrm{MeV}$. Consequently, alternative interpretations were put forward. These include conjectures that chemical equilibrium is not reached by dynamical equilibration among constituents but rather is a generic fingerprint of hadronization [60,61], or is a feature of the excited QCD vacuum [62]. The situation for $e^{+} e^{-}$collisions is, however, quite different from that for nucleus-nucleus collisions: (i) strangeness is not saturated, and (ii) additional, nonstatistical quantities such as the number of charm and beauty pairs in the system play crucial roles in the description. We also remark that the mechanism put forward in [15] is not equivalent to 'cooking' of hadrons in a hot and dense hadronic medium. Rather, the phase transition plays the driving role. In that sense, the schematic picture of [60 61] may not be so different from the mechanism worked out in [15].

These arguments enforce the conclusion that the hadro-chemical freeze-out parameters probe experimentally in an unique manner the critical line of the QCD phase transition between hadrons and QGP [63]. Our results imply that the phase boundary is reached already for the SPS beam energy of $40 \mathrm{~A} \mathrm{GeV}$. Based on the LQCD results of Fodor and Katz [43] shown in Fig. 3, the experimental freeze-out points at SPS are located in the vicinity of a critical point found in this calculation. It was pointed out recently [64,65] that the existence of a critical point for $\mu_{b}<500 \mathrm{MeV}$ requires a fine tuning of the (light) quarks masses within $5 \%$. However, it is important to recognize that serious open problems of LQCD [64,65] need to be solved before one could address such a delicate possibility and any connection between data and a possible critical point remains tenuous at best.

In any case, the results discussed above imply that light $(\mathrm{u}, \mathrm{d}, \mathrm{s})$ quarks hadronize in a scheme which is goverend by thermal weights determined by characteristic parameters $T$ and $\mu_{b}$ of the fireball formed in ultra-relativistic nuclear collisions. Due to the chemical equilibrium reached at this point, also all light quark abundances must be thermal.

We have noted above that chemical equilibrium is very likely not reached for heavy quarks. Our conjecture which led to the statistical hadronization model discussed next is that, while heavy quark abundances are manifestly non-thermal but are determined mostly by hard scattering processes, their subsequent hadronization is again governed by the thermal weights at $T$ and $\mu_{b}$, i.e. at or very near the phase boundary.

\section{The statistical hadronization model}

The statistical hadronization model (SHM) [24,26,27,28,29,31,32] assumes that the charm quarks are produced in primary hard collisions and that their total number $N_{c}$ stays 
constant until hadronization. The constancy of $N_{c}$ has been investigated in detail in [31] where it is demonstrated that variations of $N_{c}$ over the course of the evolution of the QGP are very small. Another important assumption is thermal equilibration in the QGP, at least near the critical temperature, $T_{c}$. For a detailed discussion of this issue see [31]. Here we note only that recent data on flow and energy loss of electrons from the decay of charmed hadrons lend support to the notion that charm quarks apparently come close to thermal equilibrium in the QGP. For a detailed discussion including possible elliptic flow of $J / \psi$ mesons see [66].

Hadronization of all heavy quarks takes place near the phase boundary (see above). The process of hadron formation for the bulk of heavy quarks 4 is then not determined by fragmentation functions obtained from jet studies [67] but, in analogy to the situation for light quarks, is again determined by weight functions obtained from a thermal ensemble with temperature $T$, baryo-chemical potential $\mu_{b}$ and volume V. Our hadronization prescription is similar to the treatment of heavy flavor hadronization in thermal descriptions of hadron production in $e^{+} e^{-}$collisions [58,59], although in such systems there is no chemical equilibration in the strangeness sector and several non-thermal quantities need to be externally introduced into the calculations. Note, however, that charmonia and bottomonia cannot be treated at all in this approach.

To introduce the strong departure from chemical equilibrium for charm quarks we introduce a charm fugacity $g_{c}$ which regulates the number of charm quarks in the thermal ensemble via the charm balance equation.

A key prediction of the statistical hadronization model is that the $\chi_{c} /(J / \psi)$ and $\psi^{\prime} /(J / \psi)$ ratios are entirely determined by the hadronization temperature $T$. At $T=164 \mathrm{MeV}$ these ratios are much smaller than what is observed in pp collisions [24], implying that in such systems charmonia are not produced via statistical hadronization. As we discuss below, current data for $\psi^{\prime} /(J / \psi)$ for already moderately central $\mathrm{Pb}-\mathrm{Pb}$ collisions at the top SPS energy are in good agreement with the statistical hadronization model prediction. We note that the $\psi^{\prime} /(J / \psi)$ ratio at RHIC and LHC energy should be identical ( $T$ does not change anymore) to the SPS result. The observation of any serious discrepancy from the predicted value would be difficult to explain within our model.

It is possible that hadrons containing bottom quarks and also bottomonia are produced in a statistical hadronization picture just like charm quark. The consequences of such a hypothesis were indeed worked out in [37,31,38]. Whether bottom quarks thermalize in a hot and dense fireball remains to be seen but upcoming experiments at the LHC will shed light on this interesting issue.

$\overline{4}$ We expect some fraction of nonthermalized partons, including heavy quarks, which would hadronize according to a, possibly modified, fragmentation function. This aspect is not covered by the approach discussed here. 


\subsection{Procedures}

In the following we briefly outline the calculational steps in our model [24,29]. A much more detailed and technical discussion can be found in [31,32] to which we refer the reader for more information. The model has the following input parameters: i) the total number of charm quarks $N_{c}$; ii) thermal characteristics of the fireball at chemical freezeout: temperature, $T$, baryo-chemical potential, $\mu_{b}$, and volume corresponding to one unit of rapidity $V_{\Delta y=1}$.

The total number of charm quarks is ideally obtained by measuring the charm production cross section in the nucleus-nucleus collision under consideration. This way effects such as thermal charm production or shadowing are taken into account explicitely. Unfortunately no such measurement exists today and in practice we use the charm production cross section as measured or calculated via perturbative QCD methods in pp collisions and extrapolate it to nucleus-nucleus collisions assuming scaling with the number of hard scatterings. Whenever available, estimates of shadowing are incorporated (see below). All calculations of charmonia also include the effect of the nuclear corona as discussed in [31]. In the overlapping tails of the nuclear density distributions, defined as regions with less than $10 \%$ of the central density, we assume the physics is the same as in pp collisions.

The charm balance equation [24], which has to include canonical suppression factors [26] whenever the number of charm quarks is not much larger than 1 , is used to determine a fugacity factor $g_{c}$ via:

$$
N_{c \bar{c}}^{d i r}=\frac{1}{2} g_{c} N_{o c}^{t h} \frac{I_{1}\left(g_{c} N_{o c}^{t h}\right)}{I_{0}\left(g_{c} N_{o c}^{t h}\right)}+g_{c}^{2} N_{c \bar{c}}^{t h} .
$$

Here $N_{c \bar{c}}^{d i r}=N_{c} / 2$ is the number of initially produced $c \bar{c}$ pairs and $I_{n}$ are modified Bessel functions. In the fireball of volume $V$ the total number of open $\left(N_{o c}^{t h}=n_{o c}^{t h} V\right)$ and hidden $\left(N_{c \bar{c}}^{t h}=n_{c \bar{c}}^{t h} V\right)$ charm hadrons is computed from their grand-canonical densities $n_{o c}^{t h}$ and $n_{c \bar{c}}^{t h}$, respectively. This charm balance equation is the implementation within our model of the charm conservation constraint expressed in eq. (4) below. The densities of different particle species in the grand canonical ensemble are calculated following the statistical model [3,611]. The balance equation (11) defines the fugacity parameter $g_{c}$ that accounts for deviations of heavy quark multiplicity from the value that is expected in complete chemical equilibrium. The yield of charmonia of type $j$ is obtained as: $N_{j}=g_{c}^{2} N_{j}^{t h}$, while the yield of open charm hadrons of type $i$ is: $N_{i}=g_{c} N_{i}^{t h} I_{1}\left(g_{c} N_{o c}^{t h}\right) / I_{0}\left(g_{c} N_{o c}^{t h}\right)$.

\subsection{On relevant time scales and medium effects}

In the original scenario of $J / \psi$ suppression via Debye screening [16] it is assumed that the charmonia are rapidly formed in initial hard collisions but are subsequently destroyed in the QGP. While it is clear that the production of a (colored) charm quark pair takes place at time $t_{c \bar{c}}=1 /\left(2 m_{c}\right) \leq 0.1 \mathrm{fm}$, the formation time of charmonium involves color neutralization and the build-up of its wave function. The relevant time scale has been 
studied early on [68,69] and is of order $1 \mathrm{fm}$. Similar arguments also apply for the production time of hadrons with open charm and we expect time scales comparable to those for charmonium.

We note that, at SPS energy where the 'melting scenario' was originally studied, this time is in the same range as the plasma formation time. At SPS and lower energies, charmonia can be formed in the pre-plasma phase and must be destroyed in the plasma if suppression by QGP is to take place.

At the collider energies of RHIC and especially LHC the plasma formation time is likely to be much shorter (comparable to $t_{c \bar{c}}$ ), implying that charmonium or even color octet states do not exist before the plasma is formed. Furthermore, the number of charm quark pairs can exceed 10 per unit rapidity. Initially, the 'collider' plasma will be hotter than $\mathrm{T}_{D}$, the temperature above which screening takes place, and no charmonia will be formed at all in the QGP. It is our view that the charm quarks will be effectively thermalized leading to an uncorrelated pool of $\mathrm{c}$ and $\bar{c}$ quarks. Once the plasma temperature falls below $\mathrm{T}_{D}$ charmonia can be formed in principle, but as is demonstrated by the studies performed in [31], their formation rate is likely to be low. Also we note that such a process would imply hadronization of charm quarks at temperatures possibly much higher than the critical temperature $T_{c}$. Considering the discussion about hadronization above this is very unlikely. Similar arguments should apply also to the hadronization of bottom quarks.

These observations lend further support to the notion, expressed explicitely in the statistical hadronization model, that all charmonia are produced by combination of charm and anticharm quarks at the phase boundary. We would like to emphasize that, in this scenario, the particular value of $\mathrm{T}_{D}$ which is much discussed in the recent literature [70,71], is not very important. Even if $T_{D}$ turns out to be significantly larger than $T_{c}$, in our view all charmonia are formed at the phase boundary.

Nevertheless, we note in passing that models combining the 'melting scenario' with the statistical hadronization picture have been proposed [27]. Alternatively, charmonium formation by coalescence in the plasma [34,35,72,73, as well as within transport model approaches [74,75] has been considered.

Another issue to be considered is the collision time $t_{\text {coll }}=2 R / \gamma_{c m}$, where $R$ is the radius of the (assumed equal mass) nuclei and $\gamma_{c m}$ is the Lorentz $\gamma$ factor of each of the beams in the center-of-mass system. At SPS and lower energies, $\gamma_{c m}<10$ and $t_{\text {coll }}>1 \mathrm{fm}$ for a central $\mathrm{Au}-\mathrm{Au}$ or $\mathrm{Pb}-\mathrm{Pb}$ collision, so collision time, plasma formation time, and charmonium formation time are all of the same order. Furthermore, the maximum plasma temperature may not exceed $\mathrm{T}_{D}$. In this situation any possibly formed charmonia may be broken up only 5 by the high energy nucleons still passing by from the collision and cold nuclear suppression needs to be carefully considered, as discussed, e.g., in [76,77]. However, we note in this context that the charm quarks resulting from such break-up processes eventually have to hadronize, which might again lead to charmonium production at the phase boundary if the charm quarks are kinetically thermalized, as is assumed

$\overline{5}$ We neglect break-up by hadronic co-movers, as unrealistic densities $\left(>1 / \mathrm{fm}^{3}\right)$ are required to break-up charmonia and furthermore the comover mechanism leads to an incorrect rapidity dependence of charmonium production, as discussed in the next section. 
in the statistical hadronization model [24]31. Consequently, our calculations, in which both charmonium formation before QGP production and cold nuclear suppression are neglected, may somewhat underestimate the charmonium production yield at SPS energies and below. In that sense the medium effects estimated below are upper limits for energies close to threshold.

At collider energies there will be yet another separation of time scales. At LHC energy, the momentum of a $\mathrm{Pb}$ nucleus is $2.76 \mathrm{TeV}$ per nucleon, leading to $p_{c m}=2.76 \mathrm{TeV}$ and $\gamma_{c m}=2940$, hence $t_{\text {coll }}<5 \cdot 10^{-3} \mathrm{fm}$. Even "wee" partons with momentum fraction 6 $x_{w}=2.5 \cdot 10^{-4}$ will pass by within a time $t_{w}=1 /\left(x p_{c m}\right)<0.3 \mathrm{fm}$, and will not destroy any charmonia since none exist at that time. We consequently expect that cold nuclear absorption will decrease from SPS to RHIC energy and should be negligible at LHC energy. First indications for this trend are visible in the PHENIX data [78].

Given the various time scales it becomes clear from the above discussion that the statistical hadronization model should become a quantitative tool to describe charmonium and open charm production at collider energies without the explicit need to take account of any charmonium or open charm hadron formation before the QGP is developed and of cold nuclear absorption effects. We note in passing that the issue of shadowing or saturation effects is of an entirely different nature: within the framework of the statistical hadronization model we need to know the rapidity density for open charm production in nucleus-nucleus collisions. Using this quantity, which of course contains shadowing or saturation effects, as input we can then provide cross sections for the production of all open and hidden charm hadrons.

\section{Statistical hadronization model confronts charmonium data at SPS and RHIC energies}

Here we compare the results of statistical hadronization model calculations with the most recent data from the SPS and RHIC. In the comparisons, the $J / \psi$ yield is either normalized to the number of charm pairs or we use the nuclear modification factor $R_{A A}^{J / \psi}$.

Here, $R_{A A}^{J / \psi}$ is defined as

$$
R_{A A}^{J / \psi}=\frac{\mathrm{d} N_{J / \psi}^{A A} / \mathrm{d} y}{N_{c o l l} \cdot \mathrm{d} N_{J / \psi}^{p p} / \mathrm{d} y}
$$

and relates the charmonium yield in nucleus-nucleus collisions to that expected for a superposition of independent nucleon-nucleon collisions. In this expression, $\mathrm{d} N_{J / \psi} / \mathrm{d} y$ is the rapidity density of the $J / \psi$ yield integrated over transverse momentum and $N_{\text {coll }}$ is the number of binary collisions for a given centrality class. The hadronization volume always covers one unit of rapidity for the calculations shown in this article.

6 We choose this value since it corresponds to a wee parton energy of approximately the binding energy of the $\mathrm{J} / \psi$ meson. Smaller $\mathrm{x}$ values are hence not relevant for the present considerations. 

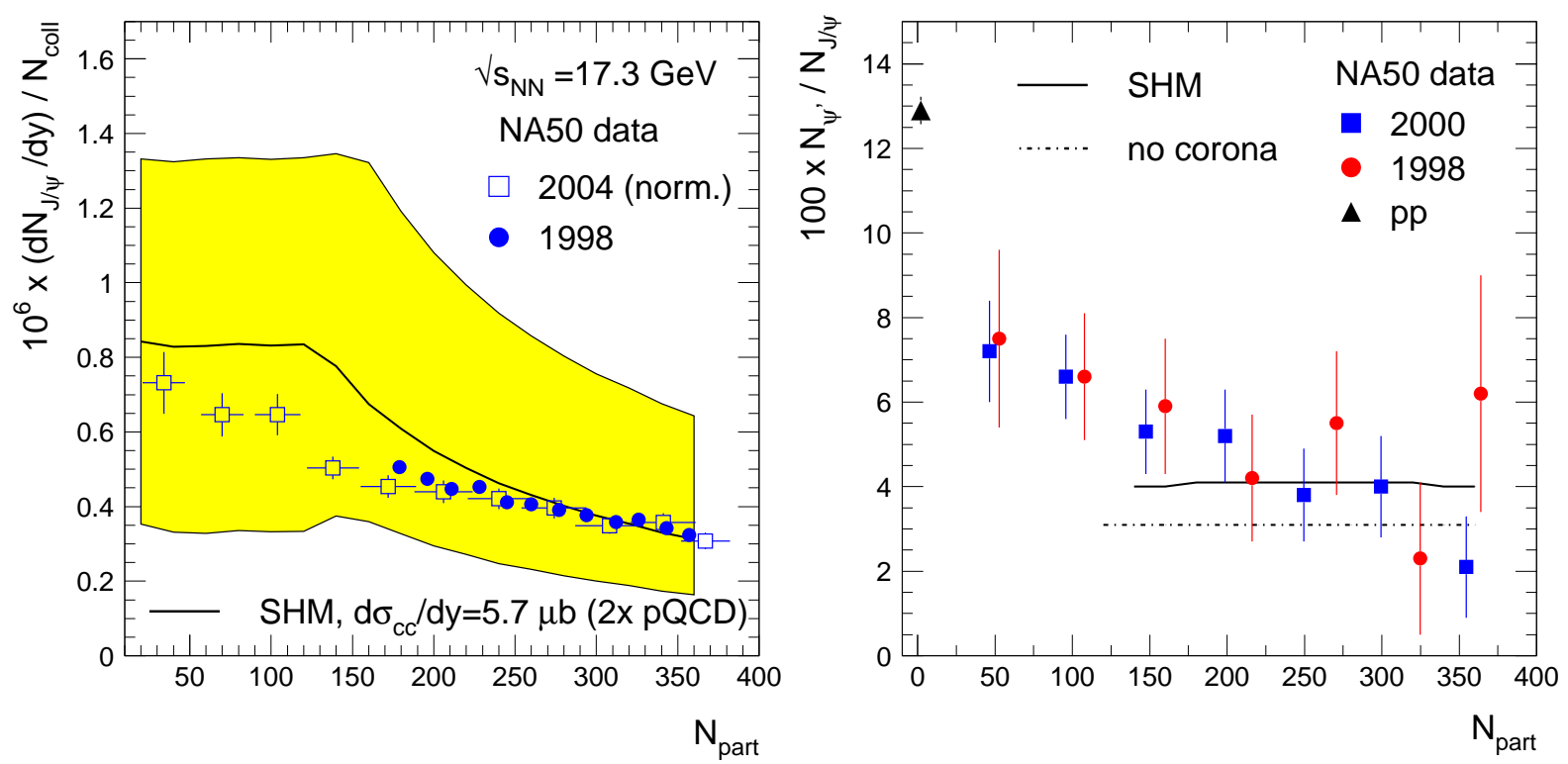

Fig. 4. Centrality dependence of $J / \psi$ yield per number of collisions (left panel) and of the ratio of yields of $\psi^{\prime}$ and $J / \psi$ (right panel) at top SPS energy. The shaded band in the left panel includes the assumed range in the charm production cross section (a factor of 2 up and down) and the error assumed for the $J / \psi$ cross section in pp collisions. The experimental data on $J / \psi$ yield are from ref. [79] (see [24]) and ref. [80] (2004 NA50 data) and on the $\psi^{\prime} / J / \psi$ ratio from ref. 8182]. Figure taken from [31].

\subsection{SPS data}

The comparison of our model calculations with the data measured at the SPS by the NA50 experiment is shown in Fig. 4.

The experimental data on $J / \psi$ production per binary collision (left panel) are well described by invoking a moderate enhancement of the charm production cross section of a factor of 2 compared to pQCD calculations [83]. New measurements by the NA60 experiment [84] indicate that the enhancement in the dimuon yield below the $J / \psi$ mass, earlier observed by NA50 [85], is of prompt origin and, as such, cannot be interpreted as an enhancement of the charm production cross section, although an experimental result on the charm cross section at this energy is currently not available. We also note that a factor of 2 enhancement is within uncertainties of the pQCD calculations.

A comparison between calculations and data [81,82] is shown in the right panel of Fig. 4 for the centrality dependence of the yield of $\psi^{\prime}$ relative to $J / \psi$. Taking account of the corona effect leads to a $25 \%$ increase of the $\psi^{\prime} /(\mathrm{J} / \psi)$ yield ratio over the value using Boltzmann factors only. Already for moderately central collisions the data approach the thermal value of the statistical hadronization model. The $\psi^{\prime} /(J / \psi)$ yield as measured in pp collisions is shown as a triangle. 


\subsection{RHIC data}

The centrality dependence of $R_{A A}^{J / \psi}$ at midrapidity as well as at more forward/backward rapidity is shown in Fig. 5. The model reproduces very well the decreasing trend versus centrality seen in the RHIC data [86] and also the relative ratio of the two rapidity intervals. Note that, in our model, the centrality dependence of the nuclear modification factor arises entirely as a consequence of the still rather moderate rapidity density of initially produced charm quark pairs at RHIC.

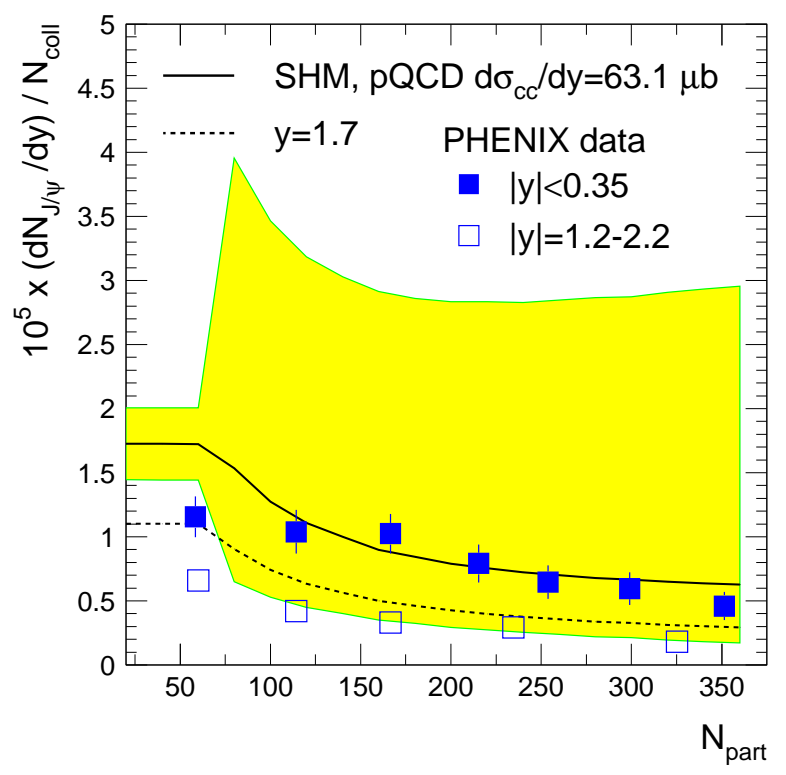

Fig. 5. Centrality dependence of the $J / \psi$ yield at midrapidity and in a more forward/backward rapidity bin per number of collisions at RHIC [86]. The experimental data are compared to model calculations, using the charm production cross section as calculated in pQCD [87]. The line is the calculation for the central value of the charm production cross section, the band corresponds to its systematic uncertainty. The dashed line is the model prediction for rapidity $y=1.7$. Figure taken from [31.

The statistical hadronization model calculations reproduce naturally the unexpected result that the observed $J / \psi$ suppression at the SPS and at RHIC are rather similar, even though the energy density of fireballs produced in central Au-Au collisions at RHIC (about $5.5 \mathrm{GeV} / \mathrm{fm}^{3}$ ) significantly exceeds that produced in $\mathrm{Pb}-\mathrm{Pb}$ collisions at SPS energy (about $3 \mathrm{GeV} / \mathrm{fm}^{3}$. Here, we think the RHIC value is conservative, since for both energies a proper time of $1 \mathrm{fm}$ was used to estimate the energy density. For a more detailed discussion see [63].

We note that the calculations shown in Fig. 5 use the charm production cross section of [87]. This is about a factor of 2 smaller than the charm cross sections derived by the PHENIX collaboration from single electron measurements [88] although that result is also consistent with the pQCD value of [87] due to the large error bands 7 . Shadowing effects may further reduce the total charm yield in nuclear collisions, see below.

Equally striking are the results on the rapidity dependence of the nuclear modification factor $R_{A A}^{J / \psi}$ observed by the PHENIX collaboration [86]. They are already visible in Fig. [5] and are explicity presented in Fig. 6. Contrary to expectations based on the assumption that $J / \psi$ suppression should scale with energy density (see, e.g., Fig. 9 in [80]), the

$\overline{7}$ Charm cross sections given by the STAR collaboration are larger by about another factor of 2 [89]. Using these in the statistical hadronization model leads to a significant overestimate of the $J / \psi$ data. 
experimentally observed suppression is smallest at midrapidity, where the energy density is largest, but increases towards forward and backward rapidities.

The observed rapidity dependence is equally at odds with predictions for charmonium suppression within the comover approach [18,19,21] since the largest comover density is at midrapidity.

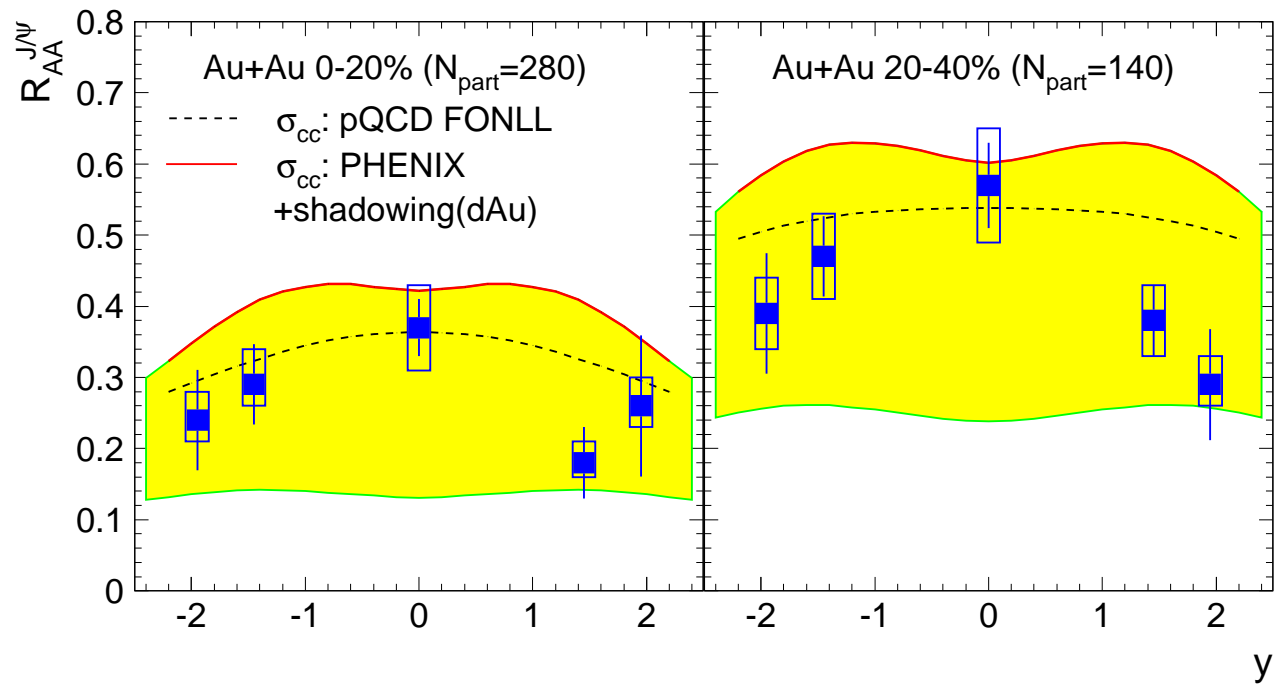

Fig. 6. Rapidity dependence of $R_{A A}^{J / \psi}$ for two centrality classes. The data from the PHENIX experiment [86] (symbols with errors) are compared to calculations (dashed and solid red lines, see text). The shaded area corresponds to calculations covering down to the lower limit (green line) of the charm cross section as measured by PHENIX [88, and using our shadowing scenario. Figure taken from [90].

A maximum in the nuclear modification factor a midrapidity emerges naturally within the framework of the statistical hadronization model. This is due to the enhanced generation of charmonium around mid-rapidity, determined by the rapidity dependence of the charm production cross section which has a maximum at midrapidity. Our calculations on the rapidity dependence of the nuclear modification factor $R_{A A}^{J / \psi}$ [90] are compared to the experimental data in Fig. 6. The dashed line in this figure is, as above, obtained using the pQCD charm production cross section [87]. The upper (red) solid line is based on the currently available experimental input into charm production at RHIC energy. It is based on the charm cross section derived by PHENIX in pp collisions [88] modified to take into account possible shadowing effects in Au-Au collisions. To this end we assume that the deviation from unity of the nuclear suppression factor in d-Au measurements [91], $R_{d A u}^{J / \psi}$, is due to shadowing and compute the shadowing factor in $\mathrm{Au}-\mathrm{Au}$ collisions as

$$
S_{A u-A u}(y)=R_{d A u}^{J / \psi}(y) R_{d A u}^{J / \psi}(-y)
$$

Using this as input into the statistical hadronization model calculation leads to a good description of the observed suppression and its rapidity dependence, as anticipated. We take this result as first evidence for the statistical generation of $\mathrm{J} / \psi$ at chemical freeze-out, as expected in the statistical hadronization model.

Nevertheless, there are alternate explanations of the RHIC data on $J / \psi$ production, as summarized recently [22]. We note in particular efforts incorporating very large gluon 
saturation effects as expected in the framework of the color-glass model [92,93] and gluon shadowing effects [94. Also the possible influence on charmonium production of a strongly coupled quark-gluon plasma has been investigated [95]. Much improved data on charmonium and open charm production will be needed to sort out these different approaches at RHIC energy. In particular, our approach of statistical hadronization for charmonium production is very sensitive to the charm cross section as the only input parameter due to the quadratic dependence. A measurement accuracy of $10 \%$ would be highly desirable and significant experimental progress it expected to come with the upgrades of PHENIX and STAR with vertex detectors.

\section{Effects of medium modifications on charmed hadron and charmonium pro- duction}

In the following, we explore charmonium and charmed hadron production and its sensitivity to medium modifications from the low energy domain near threshold $\left(\sqrt{s_{N N}} \approx 6 \mathrm{GeV}\right)$ to top SPS and RHIC energies. The lower part of this energy range can be investigated in the CBM experiment [96] at the future FAIR facility. One of the motivations for such studies was the expectation [96,97] to provide, by a measurement of D-meson production near threshold, information on their possible modification near the phase boundary. Here we demonstrate that, because of the relevant mass and time scales involved, medium effects on D-meson production are likely to be very small. Furthermore, because of the dominance of associated charm production at low energies, it turns out to be important to measure in addition to D-meson production also the yield of charmed baryons to get a good measure of the total charm production cross section. Most of the arguments presented below are not a strong function of energy and apply to higher energies as well. Possible effects due to the charm threshold at energies below $\sqrt{s_{N N}} \approx 8 \mathrm{GeV}$ are neglected. Our review is based on the recent work of [41].

For an investigation of charmonium production below top SPS energy we need an estimate of the energy dependence of the total number of produced charm quarks $N_{c}$. As no information on the charm production cross section is available for energies below $\sqrt{s_{N N}}=15$ $\mathrm{GeV}$, we have to rely on extrapolation. As basis for this extrapolation we take the energy dependence of the total charm production cross section calculated in ref. [83], as shown in Fig. 7 8. The extrapolated curves for charm production cross section are shown as solid lines in Fig. 7. Also shown for comparison are calculations with PYTHIA [98].

The total charm production cross section has an energy dependence similar to that measured for $J / \psi$ production by the HERA-B collaboration [99], also shown in Fig. 7. The extrapolation procedure for the low-energy part of the cross section obviously implies significant uncertainties. We emphasize, however, that the most robust predictions of our model, i.e. the yields of charmed hadrons and charmonia relative to the initially produced $c \bar{c}$ pair yield are not influenced by the details of this extrapolation.

8 We have rescaled these calculations to more recent values calculated at $\sqrt{s_{N N}}=200 \mathrm{GeV}$ in FONNL in ref. [87. 


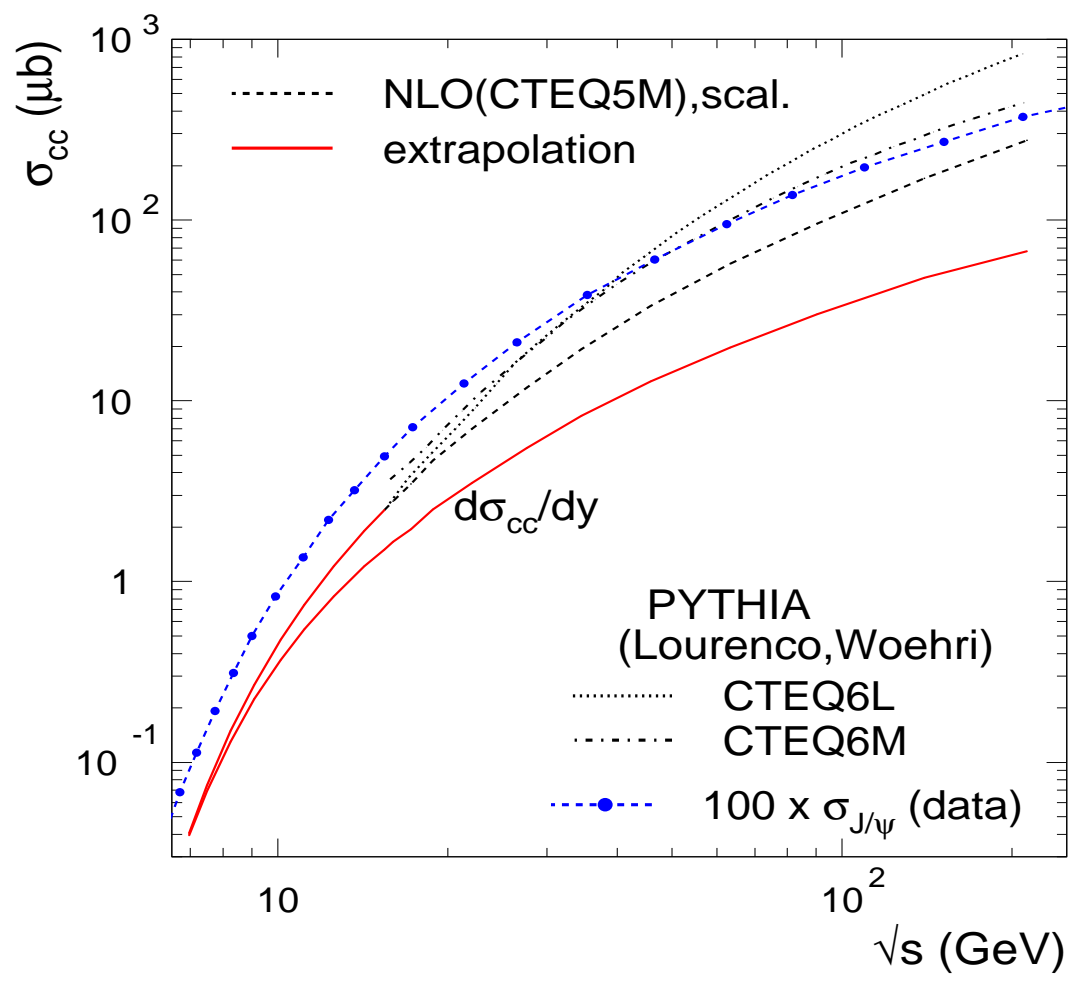

Fig. 7. Energy dependence of the charm production cross section in pp collisions. The NLO pQCD values of Vogt [83] are compared to calculations using PYTHIA, taken from ref. [98. Our extrapolations for low energies are shown as solid lines, for total and midrapidity $\left(\mathrm{d} \sigma_{c \bar{c}} / \mathrm{d} y\right)$ cross sections. The line marked with (blue) dots indicates a parameterization of the measured energy dependence of the $J / \psi$ production cross section [99].

\subsection{Charm conservation equation}

Charm conservation is essential for the discussion of possible in-medium changes of charmed hadrons and their effect on the production cross sections. We start the discussion by recalling that

$$
\sigma_{c \bar{c}}=\frac{1}{2}\left(\sigma_{D}+\sigma_{\Lambda_{c}}+\sigma_{\Xi_{c}}+\ldots\right)+\left(\sigma_{\eta_{c}}+\sigma_{J / \psi}+\sigma_{\chi_{c}}+\ldots\right)
$$

because of charm conservation. As shown in [31, annihilation of charm quarks can be neglected. In the above equation, $\sigma_{D}$ is the total cross section for the production of any D-meson. As discussed above, the cross section $\sigma_{c \bar{c}}$ is governed by the mass of the charm quark $m_{c} \approx 1.3 \mathrm{GeV}$ [67]. Consequently, perturbative QCD procedures can be applied even at low energy. Since charm production is a 'hard process' we expect no medium effects on the left-hand side of eq. 4 and, hence, there are no medium effects on the hadronic side of eq. 4 either. Such a separation of scales is not possible for strangeness production, and the situation there is not easily comparable. This also implies that there is no clear relation between near-threshold kaon production at SIS18 energy and near threshold D-meson production at FAIR. 
The much later formed D-mesons, or other charmed hadrons, may well change their mass in the hot medium. The results of various studies on in-medium modification of charmed hadrons masses [97,100,101,102,103,104,105, 106] are sometimes contradictory and we refer to [41] for a detailed assessment.

Whatever the medium effects may be, they can, because of the charm conservation equation above, only lead to a redistribution of charm quarks among the various hadronic final states. In particular, if the masses of all D-mesons are lowered by the same amount at the phase boundary, this effect would practically not be visible in the D-meson yield. Although the charm conservation equation above is strictly correct only for the total cross section we expect, within the framework of the statistical hadronization model, also little influence due to medium effects on distributions in rapidity. This is due to the fact that the crucial input into our model is $\mathrm{d} N_{c \bar{c}}^{A u A u} / \mathrm{d} y$ and there is no substantial D-meson rescattering after formation at the phase boundary. Modification of D-meson masses at the phase boundary will, however, influence the production rates for charmonia: after lowering of their masses the D-mesons will "eat away" the charm quarks which might form charmonia but since the D-mesons are much more abundant, their own yield will hardly change.

Another caveat is that medium modifications are only visible within our approach if the charmed hadrons have changed masses at chemical freeze-out. We note that excellent fits of the common (non-charmed) hadrons to predictions of the thermal model have been obtained using vacuum masses [3,6,11]. In order to see the maximum possible effect of in-medium modification, we neglect this caveat in the following and compute thermal weights for charmed hadrons with masses different from their vacuum values.

Much of the above argument about medium effects is essentially model-independent and applies equally well at all energies. Here we will consider various types of scenarios for medium modifications and study their effect within the statistical hadronization framework in the energy range from charm threshold to collider energies.

\subsection{Results}

As a first result we present, in Fig. 8, the situation for vacuum masses. The upper panel shows our predictions for the energy dependence of rapidity densities for various charmed hadrons, while in the bottom we have normalized to the number of initially produced pairs $c \bar{c}$. Beyond the generally decreasing trend for all yields with decreasing beam energy one notices first a striking behavior of the production of $\Lambda_{c}$ baryons: their yield exhibits a much weaker energy dependence than observed for other charmed hadrons. This is a result of the increase in baryo-chemical potential with decreasing energy. A similar behavior is seen for the $\Xi_{c}^{+}$baryon. In detail, the production yields of D-mesons depend also on their quark content.

We note that the model prediction of yields relative to $c \bar{c}$ pairs is a robust result, as it is to first order independent of the poorly known absolute charm production cross section. The results shown in Fig. 8 and Fig. 9 could be extrapolated up the LHC energy without visible change due to the expected unchanged temperature[107]. 


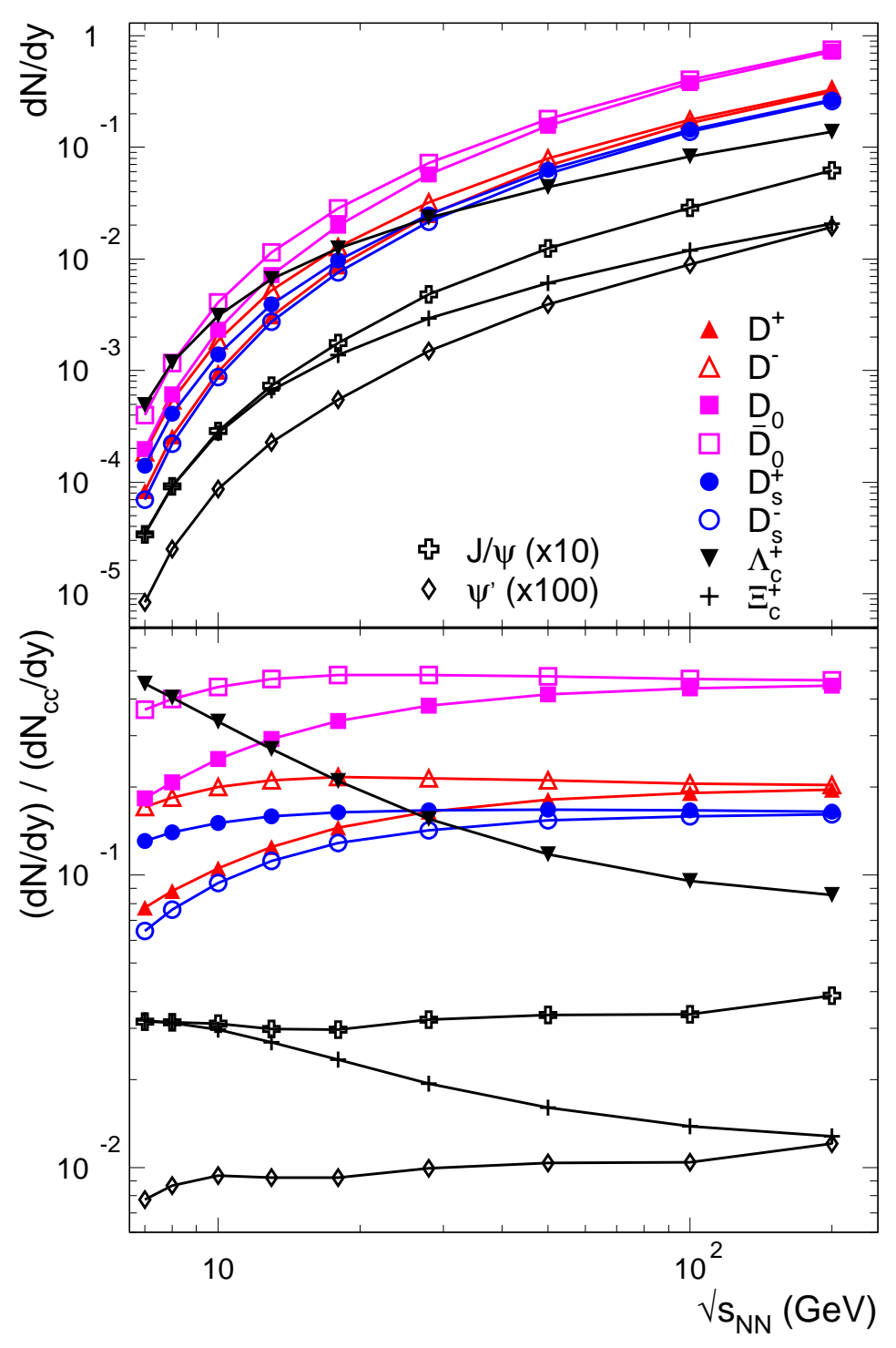

Fig. 8. Energy dependence of charmed hadron production at midrapidity computed with the statistical hadronization model. Upper panel: rapidity densities, lower panel: rapidity densities relative to the number of $c \bar{c}$ pairs. Note the scale factors of 10 and 100 for $J / \psi$ and $\psi^{\prime}$ mesons, respectively. Figure taken from [41].

The results expected if one introduces mass changes are presented in Fig. 9 for three different scenarios. In this figure we demonstrate that the total open charm yield (sum over all charmed hadrons) exhibits essentially no change if one considers mass shifts, while the effect is significant on charmonia. This is to be expected from eq. (1): as D-meson and $\Lambda_{c}$-baryon masses are reduced, the charm fugacity $g_{c}$ is changed accordingly to conserve charm. Since the $\mathrm{D}$-meson and $\Lambda_{c}$-baryon yields vary linearly with $\mathrm{g}_{c}$ we expect little change, while yields of charmonia vary more strongly, since their yields are proportional to $g_{c}^{2}$.

Note that the reduction of the $J / \psi$ yield in our model is quite different from that assumed in [72,103, 102,105, where a reduction in D-meson masses leads to (in-medium) decay of $\psi^{\prime}$ and $\chi_{c}$ into $D \bar{D}$. 


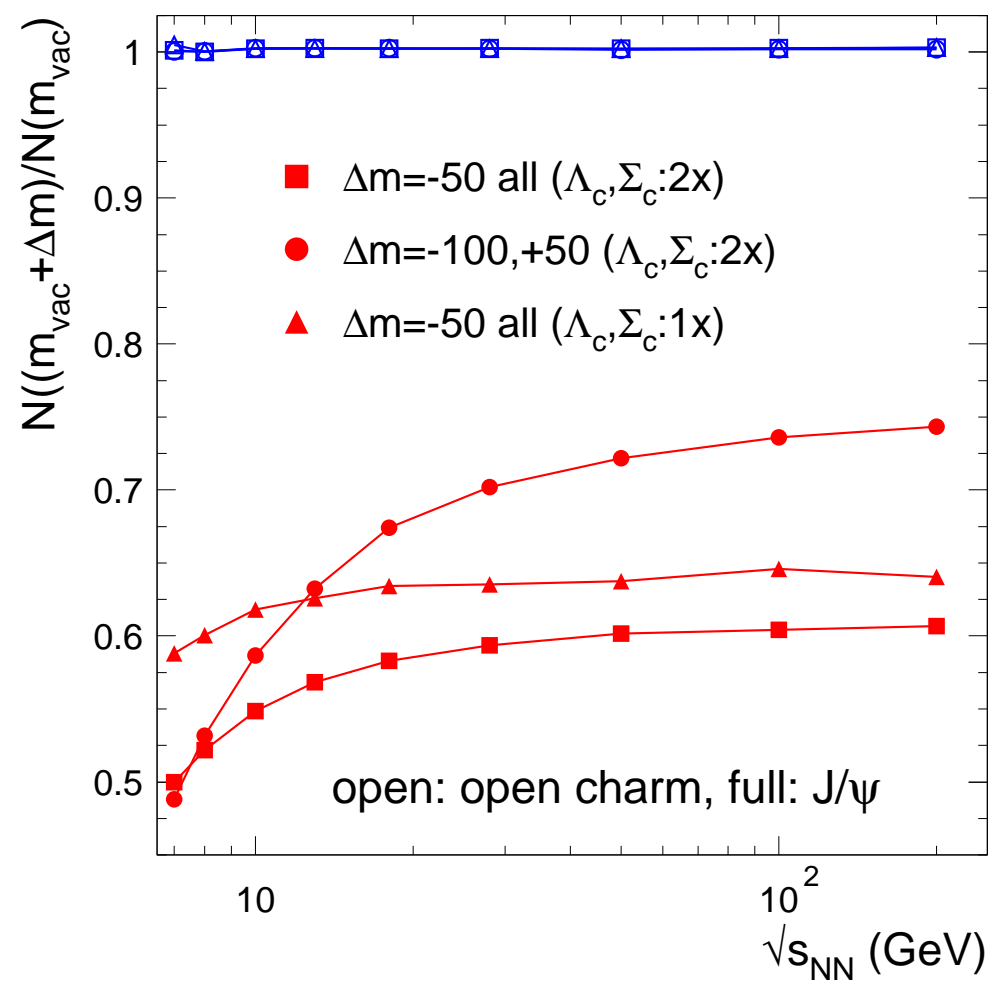

Fig. 9. Energy dependence of the relative change in the yield of open charm hadrons and of $J / \psi$ meson considering different scenarios for in-medium mass modifications. The squares correspond to a lowering in mass by $50 \mathrm{MeV}$ for charmed hadrons except for $\Lambda_{c}$ and $\Sigma_{c}$ where double the shift is assumed. The circles assume a shift downward for hadrons with charm quarks and upwards for hadrons with anticharm quarks and again double the change for $\Lambda_{c}$ and $\Sigma_{c}$. The triangles correspond to a downward shift in mass of $50 \mathrm{MeV}$ for all charmed hadrons. Figure taken from [41.

\section{Charmonium production at LHC energy}

Here we present our predictions [31,32,90,107] for charmonium production at LHC energy. At LHC energy the charm production cross section (including shadowing in $\mathrm{PbPb}$ collisions [83]) is expected to be about an order of magnitude larger than the value measured at RHIC . With $\frac{\mathrm{d} N_{c \bar{c}}^{P b P b}}{\mathrm{~d} y} \geq 10$ in central collisions canonical effects in the thermal charm calculation are expected to be small (see section 3.1) implying from eq. (11) that the charm fugacity $g_{c} \propto N_{c}$ and, hence, charmonium production will scale quadratically with $N_{c}$, at least for central collisions. As a result, the nuclear modification factor for charmonium production should exhibit a trend as function of centrality which is qualitatively different from results observed at RHIC: Depending on the actual charm production cross section $R_{A A}^{J / \psi}$ should be flat or increase with increasing number of participants $N_{\text {part }}$, i.e. as the collisions get more central. For an actual charm production cross section above $640 \mu \mathrm{b}$ $R_{A A}^{J / \psi}$ should even exceed unity for central collisions. 

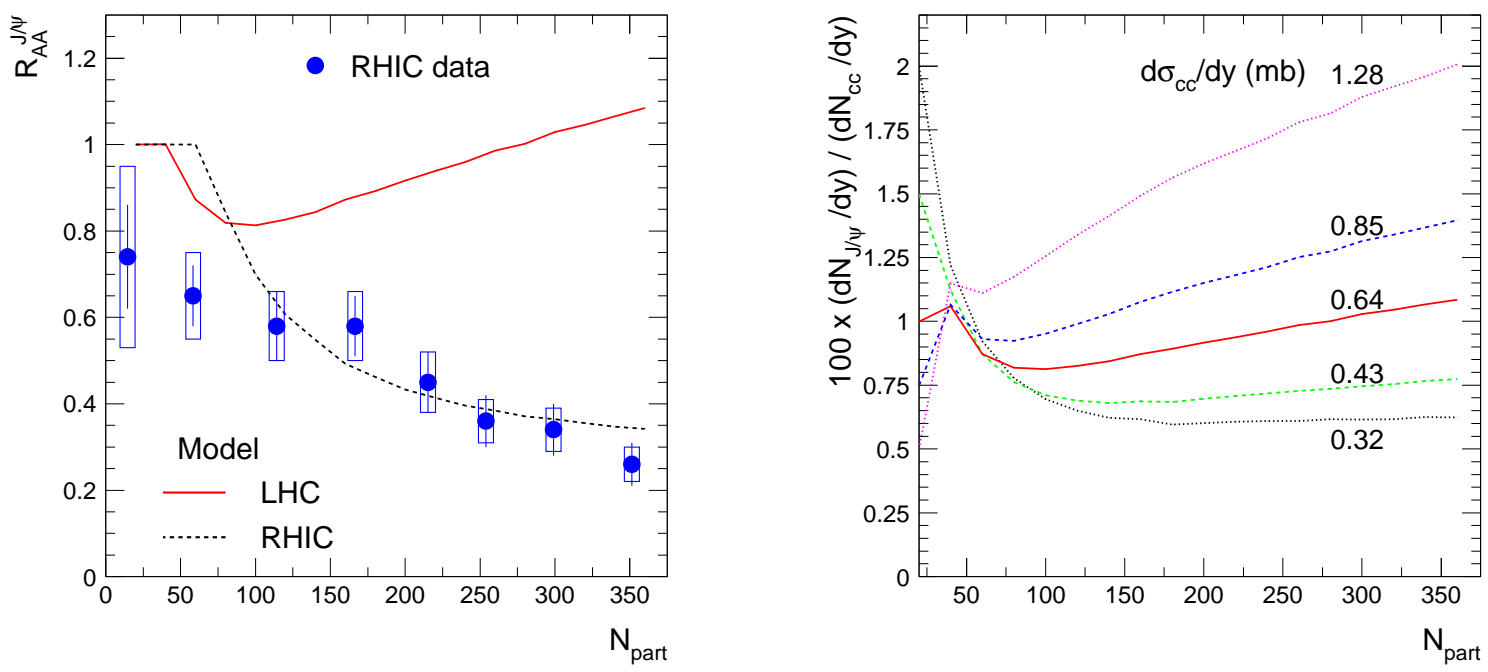

Fig. 10. Centrality dependence of $R_{A A}^{J / \psi}$ for RHIC and LHC energies (left panel) and of the $J / \psi$ rapidity density at midrapidity at LHC relative to the number of initially produced $c \bar{c}$ pairs (right panel, curves labelled by the $\mathrm{d} \sigma_{c \bar{c}} / \mathrm{d} y$ ). Figure taken from [90].

This is shown in Fig,10, In the left panel the centrality dependence of $R_{A A}^{J / \psi}$ at LHC energy is calculated using charm cross sections predicted in [83], while the comparison to charmonium data from RHIC is done using the cross section computed for pp collisions by [87] and scaled with the number of hard collisions (see section 4.2). The dramatic difference between RHIC results and LHC predictions is obvious. We note, however, that the degree of charmonium suppression or enhancement in $\mathrm{PbPb}$ collisions at $\mathrm{LHC}$ energy depends strongly on the open charm cross section. This is demonstrated in the right panel of Fig. 10, where we show the centrality dependence of $J / \psi$ production for a reasonable range in the number of initially produced $c \bar{c}$ pairs 9 . Over this range of charm cross section the yield of charmonia could vary by more than a factor of 10 and it is clear from this figure that a reasonably precise measurement of the open charm cross section in $\mathrm{PbPb}$ collisions is required to settle quantitatively the issue of statistical hadronization. The observation of an increase with $N_{\text {part }}$ of the charmonium yield will, however, be already a strong indication that charmonium production through combination of charm and anticharm quarks at the phase boundary is observed. This would be a spectacular fingerprint of deconfined and thermalized charm quarks in the quark-gluon plasma.

\section{Summary and outlook}

We have reviewed the physical basis for a description of charmonium production in ultrarelativistic nuclear collisions within the framework of the statistical hadronization model. Starting from an analysis of thermal production of hadrons composed of light $(\mathrm{u}, \mathrm{d}, \mathrm{s})$ quarks we have presented the by now convincing evidence that these hadrons are formed (nearly) simultaneously at the QCD phase boundary by a process called "chemical freezeout" with hadronic abundances quantitatively described by an equilibrated statistical ensemble. In other words, hadronization of light quarks takes place during the phase

9 Note that $R_{A A}^{J / \psi} N_{p p}^{J / \psi} / N_{p p}^{c \bar{c}}=N_{A A}^{J / \psi} / N_{A A}^{c \bar{c}}$. 
transition from the quark-gluon plasma to hadronic matter.

Heavy quarks, such as charm and bottom quarks, are not produced in the thermal fireballs formed in nucleus-nucleus collisions at high energy, but result dominantly from initial, hard collisions. Owing to their large mass which exceeds the QCD phase transition temperature by more than a factor of 5 for charm quarks and 20 for bottom quarks, chemical equilibrium is never attained for heavy quarks. However, there is by now mounting evidence from studies of charm quark flow and energy loss, that thermal equilibrium is reached in the charm quark sector. This also implies that charm quarks can travel significant distances to combine with uncorrelated anti-charm quarks. Experimental confirmation of predictions from the statistical hadronization model would then also imply direct information on the degree of deconfinement reached in the fireball.

An interesting and completely open question is whether thermal equilibrium is also reached for bottom quarks. Measurements at the LHC of possible flow and energy loss of identified bottom quarks will be crucial to shed more light on this issue.

We have described the development of the statistical hadronization model for heavy quarks and its application to charmonium production. In this model, all charm quarks are produced non-thermally but, in close analogy to the situation in the light quark sector, hadronize at the QCD phase boundary. Open and hidden charm hadrons are then formed with relative abundances again determined by statistical weight factors which can be computed again using a statistical ensemble as for the light quarks. We note that hadronization of heavy quarks at temperatures higher than the QCD critical temperature is not compatible with this view. Analyses of data on charmonium production at SPS and RHIC energies are in good agreement with predictions from the statistical hadronization model. Further, more detailed tests at RHIC energy and, in particular, analysis of data at LHC energy are essential to identify uniquely the underlying physics.

Using the charm sector to search for possible medium effects, i.e. mass modifications of charmed hadrons in the hot fireball, is severely constrained by charm conservation. This implies, e.g., that cross sections for D-meson production are very insensitive to the actual mass of D-mesons since medium modifications will modify the distribution of charm quarks among the different hadron species while the initial charm production process is governed by the mass of the charm quark.

A crucial aspect of the statistical hadronization model and, in our opinion, of all models on charmonium production in ultra-relativistic nuclear collisions is to take account of the time sequence of events from the production of charm quarks to the formation of charmonia. We have discussed this issue in detail. In particular, the formation time of open and hidden charm hadrons is important and, especially at collider energies, likely longer than the collision time or QGP formation time. This provides further support for the notion of late charmonium production at the phase boundary.

A key feature of the statistical hadronization approach is the scaling of charmonium with the square of the number of charm quark pairs in the fireball. We have argued that first traces of this scaling are already visible in the RHIC data on charmonium production. Data from the LHC will provide a crucial test for this approach. 


\section{Acknowledgments}

The authors would like to recognize the continued and very fruitful collaboration with A. Andronic and K. Redlich, with whom many of the ideas and results discussed in this review have been worked out. pbm acknowledges the support of the German Helmholtz Society through its alliance program.

\section{References}

[1] P. Braun-Munzinger, J. Stachel, J.P. Wessels and N. Xu, Phys. Lett. B 344 (1995) 43 arXiv:nucl-th/9410026] and 365 (1996) 1 nucl-th/9508020.

[2] J. Cleymans, D. Elliott, H. Satz, and R.L. Thews, Z. Phys. C 74 (1997) 319 nucl-th/9603004.

[3] P. Braun-Munzinger, I. Heppe and J. Stachel, Phys. Lett. B 465 (1999) 15 nucl-th/9903010.

[4] J. Cleymans and K. Redlich, Phys. Rev. C 60 (1999) 054908 [nucl-th/9903063].

[5] F. Becattini, J. Cleymans, A. Keranen, E. Suhonen, and K. Redlich, Phys. Rev. C 64 (2001) 024901 hep-ph/0002267.

[6] P. Braun-Munzinger, D. Magestro, K. Redlich, and J. Stachel, Phys. Lett. B 518 (2001) 41 hep-ph/0105229].

[7] N. Xu and M. Kaneta, Nucl. Phys. A 698 (2002) 306c.

[8] F. Becattini, J. Phys. G 28 (2002) 1553.

[9] R. Rapp and E. Shuryak, Phys. Rev. Lett. 86 (2001) 2980 hep-ph/0008326.

[10] F. Becattini, M. Gazdzicki, J. Manninen, Phys. Rev. C 73 (2006) 044905 hep-ph/0511092].

[11] A. Andronic, P. Braun-Munzinger, J. Stachel, Nucl. Phys. A 772 (2006) 167 nucl-th/0511071.

[12] J. Manninen, F. Becattini, Phys. Rev. D78 (2008) 054901 arXiv:0806.4100 [nucl-th].

[13] P. Braun-Munzinger, K. Redlich, and J. Stachel, invited review in Quark Gluon Plasma 3, eds. R.C. Hwa and X.N. Wang, (World Scientific Publishing, 2004) nucl-th/0304013.

[14] A. Andronic, P. Braun-Munzinger, J. Stachel, Phys. Lett. B submitted, arXiv:0812.1186 [nucl-th].

[15] P. Braun-Munzinger, J. Stachel, C. Wetterich, Phys. Lett. B 596 (2004) 61 nucl-th/0311005.

[16] T. Matsui, H. Satz, Phys. Lett. B 178 (1986) 416.

[17] M.C. Abreu et al., NA50 collaboration, Phys. Lett. B 499 (2001) 85.

[18] S. Gavin, R. Vogt, Phys. Rev. Lett. 78 (1997) 1006. 
[19] A. Capella, A. B. Kaidalov, D. Sousa, Phys. Rev. C 65 (2002) 054908.

[20] T.Barnes, E.S. Swanson, C.Y. Wong, X.M. Xu, Phys. Rev. C 68 (2003) 014903 nucl-th/0302052.

[21] C. Spieles et al., Phys. Rev. C 60 (1999) 054901.

[22] R. Granier de Cassagnac, J. Phys. G35 (2008) 104023, arXiv:0806.0046 [hep-ph].

[23] M. Gazdzicki, M. Gorenstein, Phys. Rev. Lett. 83 (1999) 4009.

[24] P. Braun-Munzinger, J. Stachel, Phys. Lett. B 490 (2000) 196 nucl-th/0007059]; Nucl. Phys. A 690 (2001) 119c [nucl-th/0012064].

[25] B.W. Zhang, C.M. Ko, W. Liu, Phys. Rev. C 77 (2008) 024901.

[26] M.I. Gorenstein, A.P. Kostyuk, H. Stöcker, W. Greiner, Phys. Lett. B 509 (2001) 277 [hep-ph/0010148]; Phys. Lett. B 524 (2002) 265 [hep-ph/0104071].

[27] L. Grandchamp, R. Rapp, Phys. Lett. B 523 (2001) 60 hep-ph/0103124; Nucl. Phys. A 709 (2002) 415 hep-ph/0205305.

[28] A.P. Kostyuk, M.I. Gorenstein, H. Stöcker, W. Greiner, Phys. Lett. B 531 (2002) 195 hep-ph/0110269]; Phys. Rev. C 68 (2003) 041902 [hep-ph/0305277].

[29] A. Andronic, P. Braun-Munzinger, K. Redlich, J. Stachel, Phys. Lett. B 571 (2003) 36 nucl-th/0303036.

[30] E.L. Bratkovskaya, A.P. Kostyuk, W. Cassing, H. Stöcker, Phys. Rev. C 69 (2004) 054903 nucl-th/0402042.

[31] A. Andronic, P. Braun-Munzinger, K. Redlich, J. Stachel, Nucl. Phys. A 789 (2007) 334 nucl-th/0611023.

[32] A. Andronic, P. Braun-Munzinger, K. Redlich, J. Stachel, Phys. Lett. B 652 (2007) 259, nucl-th/0701079.

[33] X. Zhao, R. Rapp, arXiv:0810.4566 [nucl-th].

[34] R.L. Thews, M. Schroedter, J. Rafelski, Phys. Rev. C 63 (2001) 054905 [hep-ph/0007323].

[35] R.L. Thews, AIP Conf. Proc. 631 (2002) 490 [hep-ph/0206179].

[36] R.L. Thews, M.L. Mangano, Phys. Rev. C 73 (2006) 014904 hep-ph/0505055].

[37] L. Grandchamp, S. Lumpkins, D. Sun, H. van Hees, R. Rapp, Phys. Rev. C 73 (2006) 064906 hep-ph/0507314.

[38] F. Becattini, Phys. Rev. Lett. 95 (2005) 022301 hep-ph/0503239.

[39] R. Rapp, D. Blaschke, P. Crochet, arXiv:0807.2470 [hep-ph].

[40] P. Bhaduri, P. Hedge, H. Satz, P. Tribedi, arXiv:0812.3856 [hep-ph].

[41] A. Andronic, P. Braun-Munzinger, K. Redlich, J. Stachel, Phys. Lett. B 659 (2007) 149, arXiv:0708.1488 [nucl-th].

[42] R. Hagedorn, Nuovo Cimento 35 (1965) 395; see also CERN-TH-4100/85 (1985).

[43] Z. Fodor, S.D. Katz, J. High En. Phys. 203 (2002) 14 hep-lat/0106002], ibid 404 (2004) 50 hep-lat/0402006. 
[44] C.R. Allton, S. Ejiri, S.J. Hands, O. Kaczmarek, F. Karsch, E. Laermann, Ch. Schmidt, L. Scorzato, Phys. Rev. D 66 (2002) 074507 hep-lat/0204010].

[45] P. de Forcrand, O. Philipsen, Nucl. Phys. B 642 (2002) 290 hep-lat/0205016.

[46] F. Karsch, J. Phys. G 31 (2005) S633 [hep-lat/0412038].

[47] Z. Fodor, PoS CPOD07 (2007) 27, arXiv:0712.2930 [hep-lat].

[48] Y. Aoki, G. Endrodi, Z. Fodor, S.D. Katz, K.K. Szabo, Nature 443 (2006) 675 hep-lat/0611014.

[49] A. Di Giacomo, arXiv:0901.0227 [hep-lat].

[50] P. Braun-Munzinger, J. Wambach, Rev. Mod. Phys. (in print), arXiv:0801.4256 [hep-ph].

[51] M. Cheng et al., Phys. Rev. D 74 (2006) 054507 hep-lat/0608013.

[52] P. Braun-Munzinger, J. Stachel, J. Phys. G 28 (2002) 1971 nucl-th/0112051 (see also P. Braun-Munzinger, nucl-ex/0508024).

[53] A. Tawfik, Phys. Rev. D 71 (2005) 054502 hep-ph/0412336.

[54] J. Cleymans, K. Redlich, Phys. Rev. Lett. 81 (1998) 5284 nucl-th/9808030]; Phys. Rev. C 60 (1999) 054908 nucl-th/9903063.

[55] P. Koch, B. Müller, J. Rafelski, Phys. Rep. 142 (1986) 167.

[56] C. Greiner, S. Leupold, J. Phys. G 27 (2001) L95.

[57] P. Huovinen, J. Kapusta, Phys.Rev. C 69 (2004) 014902 [nuclth/0310051].

[58] A. Andronic, F. Beutler, P. Braun-Munzinger, K. Redlich, J. Stachel, arXiv:0804.4132 [hep$\mathrm{ph}]$.

[59] F. Becattini, P. Castorina, J. Manninen, H. Satz, arXiv:0805.0964 [hep-ph].

[60] R. Stock, Phys. Lett. B 456 (1999) 277 hep-ph/9905247.

[61] U.W. Heinz, Nucl. Phys. A661 (1999) 140 [nucl-th/9907060].

[62] P. Castorina, D. Kharzeev, H. Satz, Eur. Phys. J. C 52 (2007) 187 arXiv:0704.1426 [hep-ph].

[63] J. Stachel, Int. J. Mod. Phys. A21 (2006) 1750 nucl-ex/0510077.

[64] O. Philipsen, PoS LAT2005 (2005) 016 hep-lat/0510077.

[65] O. Philipsen, PoS CPOD07 (2007) 28, arXiv:0710.1217 [hep-ph].

[66] D. Krieg, M. Bleicher, arXiv:0806.0736 [nucl-th].

[67] W.-M. Yao et al., J. Phys. G 33 (2006) 1 http://pdg.1bl.gov/.

[68] F. Karsch and R. Petronzio, Phys. Lett. B 193 (1987) 105.

[69] J.-P. Blaizot and J.-Y. Ollitrault, Phys. Rev. D 39 (1989) 232.

[70] F. Karsch, D. Kharzeev, H. Satz, Phys. Lett. B 637 (2006) 75 hep-ph/0512239]; H. Satz, Nucl. Phys. A 783 (2007) 249 [hep-ph/0609197.

[71] A. Mocsy, P. Petrecky, Phys. Rev. D 77 (2008) 014501 arXiv:0705.2559 [hep-lat]; Phys. Rev. Lett. 99 (2007) 211602 arXiv:0706.2183 [hep-ph]. 
[72] L. Grandchamp, R. Rapp, G.E. Brown, Phys. Rev. Lett. 92 (2004) 212301 hep-ph/0306077].

[73] L. Yan, P. Zhuang, N. Xu, Phys. Lett. B 607 (2005) 107 nucl-th/0411093]; Phys. Rev. Lett. 97 (2006) 232301 nucl-th/0608010.

[74] B. Zhang, C.M. Ko, B.A. Li, Z.W. Lin, S. Pal, Phys. Rev. C 65 (2002) 054909 nucl-th/0201038.

[75] E.L. Bratkovskaya, W. Cassing, H. Stöcker, Phys. Rev. C 67 (2003) 054905 nucl-th/0301083]; O. Linnyk, E. L. Bratkovskaya, W. Cassing, H. Stöcker, Nucl. Phys. A 786 (2007) 183 nucl-th/0612049.

[76] H. Satz, J. Phys. G 32 (2006) R25 hep-ph/0512217.

[77] F. Arleo, V. Tram, Eur. Phys. J. C55 (2008) 449 [hep-ph/0612043].

[78] S.S. Adler et al. (PHENIX), Phys. Rev. Lett. 96 (2006) 012304 nucl-ex/0507032.

[79] J. Gosset, A. Baldisseri, H. Borel, F. Staley, Y.Terrien, Eur. Phys. J. C 13 (2000) 63 nucl-ex/9906002.

[80] B. Alessandro et al. (NA50), Eur. Phys. J. C 39 (2005) 335 hep-ex/0412036.

[81] B. Alessandro et al. (NA50), Eur. Phys. J. C49 (2007) 559 nucl-ex/0612013.

[82] M.C. Abreu et al. (NA50), Phys. Lett. B 466 (1999) 408.

[83] R. Vogt, Int. J. Mod. Phys. E 12 (2003) 211 hep-ph/0111271.

[84] R. Shahoyan et al. (NA60), Nucl. Phys. A 774 (2006) 677c.

[85] M.C. Abreu et al. (NA50), Nucl. Phys. A 698 (2002) 539c.

[86] A. Adare et al. (PHENIX),Phys. Rev. Lett. 98 (2007) 232301 nucl-ex/0611020].

[87] M. Cacciari, P. Nason, R. Vogt, Phys. Rev. Lett. 95 (2005) 122001 hep-ph/0502203.

[88] A. Adare et al. (PHENIX), Phys. Rev. Lett. 97 (2006) 252002 hep-ex/0609010.

[89] J. Adams et al. (STAR), Phys. Rev. Lett. 94 (2005) 062301 nucl-ex/0407006.

[90] A. Andronic, P. Braun-Munzinger, K. Redlich, J. Stachel, J. Phys. G 35 (2008) 104155 arXiv:0805.4781 [nucl-th].

[91] S.S. Adler et al. (PHENIX), Phys. Rev. C 71 (2005) 034908 nucl-ex/0409015.

[92] D. Kharzeev, M. Levin, M. Nardi, K. Tuchin, arXiv:0808.2954 [hep-ph].

[93] D. Kharzeev, M. Levin, M. Nardi, K. Tuchin, arXiv:0809.2933 [hep-ph].

[94] L. Bravina, A. Capella, E.G. Ferreiro, A.B. Kaidalov, K. Twoniuk, E. Zabrodin, arXiv:0811.0790 [hep-ph].

[95] C. Young, E. Shuryak, arXiv:0803.2866 [nucl-th].

[96] P. Senger, J. Phys. Conf. Series 50 (2006) 357.

[97] L. Tolos, J. Schaffner-Bielich, H. Stöcker, Phys. Lett. B 635 (2006) 85 [nucl-th/0509054.

[98] C. Lourenço, H. Wöhri, Phys. Rept. 433 (2006) 127 hep-ph/0609101. 
[99] I. Abt et al. (HERA-B), Phys. Lett. B 638 (2006) 407 hep-ex/0512029.

[100] K. Tsushima, D.H. Lu, A.W. Thomas, K. Saito, R.H. Landau, Phys. Rev. C 59 (1999) 2824 [nucl-th/9810016].

[101] A. Sibirtsev, K. Tsushima, A.W. Thomas, Eur. Phys. J. A 6 (1999) 351 nucl-th/9904016.

[102] A. Sibirtsev, K. Tsushima, K. Saito, A.W. Thomas, Phys. Lett. B 484 (2000) 23 nucl-th/9904015.

[103] A. Hayashigaki, Phys. Lett. B 487 (2000) 96 nucl-th/0001051.

[104] W. Cassing, E.L. Bratkovskaya, A. Sibirtsev, Nucl. Phys. A 691 (2001) 753 nucl-th/0010071.

[105] B. Friman, S.H. Lee, T. Song, Phys. Lett. B 548 (2002) 153 nucl-th/0207006].

[106] M.F.M. Lutz, C.L. Korpa, Phys. Lett. B 633 (2006) 43 nucl-th/0510006].

[107] A. Andronic, P. Braun-Munzinger, J. Stachel, in N. Armesto et al., J. Phys. G 35 (2008) 054001 arXiv:0711.0974 [hep-ph]. 